FUNCTION SPACES XII

BANACH CENTER PUBLICATIONS, VOLUME 119

INSTITUTE OF MATHEMATICS

POLISH ACADEMY OF SCIENCES

WARSZAWA 2019

\title{
TURING PATTERNS, LENGYEL-EPSTEIN SYSTEMS AND FABER SPLINES
}

\author{
HANS TRIEBEL \\ Institut für Mathematik, Friedrich-Schiller-Universität \\ D-07737 Jena, Germany \\ E-mail: hans.triebel@uni-jena.de
}

\begin{abstract}
This paper deals with the Lengyel-Epstein CIMA (chlorite-iodide-malonic acid) system of non-linear parabolic equations in the context of function spaces, especially of HölderZygmund type. We discuss in particular the size of Turing patterns (if occur) in dependence on initial data. This will be based on expansions in terms of Faber splines.
\end{abstract}

1. Introduction and motivation. A. M. Turing (1912-1954), well known for his significant contributions to the theory of computing, artificial intelligence, cryptography and for cracking the code used by the German Enigma machine in World War II, dealt at the end of his short life with mathematical biology. In his seminal paper 30. (now by far his most quoted publication) he developed a theory that competing chemicals are responsible for plant evolution and pattern creation. The beginning of the Abstract of his paper reads as follows.

It is suggested that a system of chemical substances called morphogens, reacting together and diffusing through a tissue, is adequate to account for the main phenomena of morphogenesis. Such a system, although it may originally be quite homogeneous, may later develop a pattern or structure due to an instability of the homogeneous equilibrium, which is triggered off by random disturbances.

Turing coined the word morphogen whereas morphogenesis (origination and development of morphological characters) and morphological (derived from morphology, the branch of biology that deals with the form of living organisms and with relations between their

2010 Mathematics Subject Classification: Primary 46E35; Secondary 35K55, 35Q92, 42B35, 92C15.

Key words and phrases: Turing patterns, Lengyel-Epstein systems, function spaces, HölderZygmund spaces, Faber splines.

The paper is in final form and no version of it will be published elsewhere. 
structure) are somewhat older. According to [11, p. 71] they go back to J. W. von Goethe (1749-1832) who developed a theory of plant evolution that hypothesizes that most plants come from one archetypal plant. (But this was not taken very seriously and Goethe was angry about this.) But it needed almost 40 years to confirm Turing's vision experimentally in [2]. A strip of gel loaded with starch indicator was fed with constant concentrations of malonic acid (MA) at one end and of chlorite $\left(\mathrm{ClO}_{2}^{-}\right)$and iodide $\left(\mathrm{I}^{-}\right)$ions at the other end. Nonuniform patterns both parallel and perpendicular to the direction along which the reactants diffuse towards each other were observed. The original five-variable model in [2] could be simplified in [6, 7] essentially resulting in

$$
\begin{aligned}
& \partial_{t} u-\sigma_{u} \Delta u=a-u-\frac{4 u v}{1+u^{2}}, \\
& \partial_{t} v-\sigma_{v} \Delta v=b c u-\frac{c u v}{1+u^{2}},
\end{aligned}
$$

where $u=u(x, t)$ and $v=v(x, t)$ denote the chemical concentrations of the activator (substance that activates) iodide $\mathrm{I}^{-}$and the inhibitor (substance which slows down, prevents reaction) chlorite $\mathrm{ClO}_{2}^{-}$at time $t>0$ and $x \in \Omega$ (underlying domain). As usual $\partial_{t}=\partial / \partial t$, whereas $\Delta$ is the Laplacian with respect to the space variables. Furthermore, $\sigma_{u}, \sigma_{v}$ are positive diffusion constants and $a, b, c$ are positive constants, reflecting feed concentrations of the chemicals involved. This is the Lengyel-Epstein CIMA system, usually studied in (smooth) bounded domains $\Omega$ in $\mathbb{R}^{n}, n \in \mathbb{N}$, with some (unimportant) boundary conditions (preferable zero Dirichlet or Neumann conditions) and given initial data

$$
u(x, 0)=u_{0}(x), \quad v(x, 0)=v_{0}(x), \quad x \in \Omega .
$$

A detailed discussion of what is meant by Turing instability and related Turing patterns may be found in [13, Chapter 7]. We refer the reader also to [11, Chapter 2] and Turing's original paper 30. In any case the question under which conditions Turing patterns may occur is a rather delicate interplay between the two diffusion coefficients $\sigma_{u}, \sigma_{v}$ (must be very different) and the positive chemical constants $a, b, c$. Some details may be found in [13, Section 7.5.2, pp. 128-130]. The question of whether systems like 11.1, , 1.2, maybe with more general non-linearities on the related right-hand sides, can produce Turing patterns depends on the following three ingredients:

1. The system under consideration, here 1.1$],(1.2)$, has a positive (at best unique) constant solution $\bar{u}, \bar{v}$, in our case

$$
\bar{u}=\frac{a}{4 b+1}, \quad \bar{v}=b\left(1+\bar{u}^{2}\right) .
$$

2. One expands the system under consideration, here $11.1,(1.2)$, with respect to $(u, v)$ at $(\bar{u}, \bar{v})$ and asks of whether the resulting linear system (neglecting higher powers of $u-\bar{u}$ and $v-\bar{v}$ ) has Turing unstable solutions of type $e^{\lambda t} u(x), e^{\lambda t} v(x)$ with $\operatorname{Re} \lambda>0$.

3. If this is the case then it is Turing's vision that under lucky circumstances the so far neglected nonlinear terms have the ability to catch these unstable solutions (of the linearized system), to prevent them to escape to infinity and to produce at the end steady non-constant positive solutions $u(x, t)=u(x)$ and $v(x, t)=v(x)$ of the related elliptic 
system

$$
\begin{aligned}
\sigma_{u} \Delta u & =\frac{4 u v}{1+u^{2}}+u-a, \\
\sigma_{v} \Delta v & =\frac{c u v}{1+u^{2}}-b c u .
\end{aligned}
$$

It comes out that this problem is a matter of the Leray-Schauder degree theory (fixed point theorem) discussing under which conditions for the parameters the system (1.5), 1.6 has non-constant positive solutions $u(x), v(x)$ and under which conditions this is not the case. Related assertions may be found in [12] and [13. Lemma 7.5, p. 129].

Turing instabilities, Turing patterns and in particular Lengyel-Epstein systems attracted a lot of attention in recent times. We refer the reader to [1, 4, 8, 9, 10, 15, 31, 32, 33] and the literature mentioned there. This covers also several generalizations and modifications of the classical Lengyel-Epstein equations to systems fractional in space and time and with more general nonlinearities.

Somewhat in contrast to the set-up and the technicalities in the above-mentioned literature we deal with the Lengyel-Epstein equations $11.1,11.2$ in the context of (weighted and unweighted) function spaces in $\mathbb{R}^{n}$. This might be considered as the continuation of our approach to the Navier-Stokes equations in [25, 26] and to the Keller-Segel equations underlying chemotaxis (the movement of biological cells or organisms in response to chemical gradients) in [28].

Section 2 deals with basic assertions, in particular the existence and uniqueness of mild and strong solutions (local in time) of 1.1, , 1.2 complemented by suitable initial data. In addition we ask for properties of these solutions being of relevance with respect to the above-described chemical background. This applies in particular to the positivity of related solutions. In Section 3 we complement the properties collected in Section 2 by the assumption that the related Lengyel-Epstein system displays Turing patterns. It has been discussed in the literature in connection with Turing instabilities how large Turing patterns, if occur, might be in dependence on initial data (and the size of related domains reflecting the size of the plant or animal). We refer the reader in particular to [11, Section 3.1, pp. 142-156], entitled 'Mammalian coat patterns - how the leopard got is spots'. The underlying equations in [11, (3.1), p. 145] which are responsible for dots and stripes on the skins of animals in the context of Turing patterns, are very much similar to the Lengyel-Epstein system (1.1), 1.2. According to [11, based on some qualitative discussions, dots and stripes originating from related Turing patterns cannot be very small (in dependence on initial data and other ingredients). It is mentioned there that the elephant is too big to have dots caused by Turing patterns. We deal with questions of this type in Section 3 and estimate how large Turing patterns must be in dependence on initial data. The related assertions in Section 3 might be considered as the main results of this paper. One may also consult [28, Section 5.6, pp. 85-93] for related discussions.

The main bulk of the paper consists of the Sections 1, 2 and Sections 3.1,3.3 inclusively where we tried to simplify the needed function spaces as much as possible. The final Section 3.4 is different. Here we indicate how future research may look like based on the full machinery of the recent theory of function spaces. It is directed to specialists in the theory of function spaces and may be skipped otherwise. 


\section{Lengyel-Epstein systems in function spaces}

2.1. Function spaces and heat kernels. We use standard notation. Thus, $\mathbb{N}$ is the collection of all natural numbers, $\mathbb{N}_{0}=\mathbb{N} \cup\{0\}$, and $\mathbb{R}^{n}$ is the Euclidean $n$-space, where $n \in \mathbb{N}$. Put $\mathbb{R}=\mathbb{R}^{1}$, whereas $\mathbb{C}$ is the complex plane. Let $S\left(\mathbb{R}^{n}\right)$ be the usual Schwartz space and $S^{\prime}\left(\mathbb{R}^{n}\right)$ be its dual, the space of all tempered distributions on $\mathbb{R}^{n}$. Further, $L_{p}\left(\mathbb{R}^{n}\right)$ with $1 \leq p \leq \infty$, is the standard Banach space in $\mathbb{R}^{n}$, normed by

$$
\left\|f \mid L_{p}\left(\mathbb{R}^{n}\right)\right\|=\left(\int_{\mathbb{R}^{n}}|f(x)|^{p} \mathrm{~d} x\right)^{1 / p}
$$

with the natural modification if $p=\infty$. As usual, $\mathbb{Z}$ is the collection of all integers; and $\mathbb{Z}^{n}$, where $n \in \mathbb{N}$, denotes the lattice of all points $m=\left(m_{1}, \ldots, m_{n}\right) \in \mathbb{R}^{n}$ with $m_{j} \in \mathbb{Z}$. Let as usual $\partial_{j}=\partial / \partial x_{j}, \partial_{j}^{m}=\partial^{m} / \partial x_{j}^{m}, m \in \mathbb{N}_{0}\left(\partial_{j}^{0} f=f\right)$ and let in particular $\Delta=\sum_{j=1}^{n} \partial_{j}^{2}$ be the Laplacian with respect to the space variable $x=\left(x_{1}, \ldots, x_{n}\right) \in \mathbb{R}^{n}$. Let $\partial_{t}=\partial / \partial t$ where $t$ stands for the time variable.

If $\varphi \in S\left(\mathbb{R}^{n}\right)$ then

$$
\widehat{\varphi}(\xi)=(F \varphi)(\xi)=(2 \pi)^{-n / 2} \int_{\mathbb{R}^{n}} e^{-i x \xi} \varphi(x) \mathrm{d} x, \quad \xi \in \mathbb{R}^{n},
$$

denotes the Fourier transform of $\varphi$. Also, $F^{-1} \varphi$ and $\varphi^{\vee}$ stand for the inverse Fourier transform, given by the right-hand side of 2.2 with $i$ in place of $-i$. Here $x \xi$ denotes the scalar product in $\mathbb{R}^{n}$. Both $F$ and $F^{-1}$ are extended to $S^{\prime}\left(\mathbb{R}^{n}\right)$ in the standard way. Define $\varphi_{0} \in S\left(\mathbb{R}^{n}\right)$ by

$$
\varphi_{0}(x)=1 \text { if }|x| \leq 1 \quad \text { and } \quad \varphi_{0}(x)=0 \text { if }|x| \geq 3 / 2,
$$

and let

$$
\varphi_{k}(x)=\varphi_{0}\left(2^{-k} x\right)-\varphi_{0}\left(2^{-k+1} x\right), \quad x \in \mathbb{R}^{n}, \quad k \in \mathbb{N} .
$$

Since

$$
\sum_{j=0}^{\infty} \varphi_{j}(x)=1 \quad \text { for } x \in \mathbb{R}^{n},
$$

the $\varphi_{j}$ form a dyadic resolution of unity. The entire analytic functions $\left(\varphi_{j} \widehat{f}\right)^{\vee}(x)$ make sense pointwise in $\mathbb{R}^{n}$ for any $f \in S^{\prime}\left(\mathbb{R}^{n}\right)$.

Definition 2.1. Let $n \in \mathbb{N}$. Let $\varphi=\left\{\varphi_{j}\right\}_{j=0}^{\infty}$ be the above resolution of unity and let $s \in \mathbb{R}$. Then $\mathcal{C}^{s}\left(\mathbb{R}^{n}\right)$ is the collection of all $f \in S^{\prime}\left(\mathbb{R}^{n}\right)$ such that

$$
\left\|f\left|\mathcal{C}^{s}\left(\mathbb{R}^{n}\right) \|_{\varphi}=\sup _{j \in \mathbb{N}_{0}, x \in \mathbb{R}^{n}} 2^{j s}\right|\left(\varphi_{j} \widehat{f}\right)^{\vee}(x) \mid\right.
$$

is finite.

REmark 2.2. These are the so-called Hölder-Zygmund spaces. They are special cases of the spaces $A_{p, q}^{s}\left(\mathbb{R}^{n}\right)$ with $A \in\{B, F\}, s \in \mathbb{R}$ and $0<p, q \leq \infty$,

$$
\mathcal{C}^{s}\left(\mathbb{R}^{n}\right)=B_{\infty, \infty}^{s}\left(\mathbb{R}^{n}\right), \quad s \in \mathbb{R} .
$$

The theory of these spaces, including $\mathcal{C}^{s}\left(\mathbb{R}^{n}\right)$ and their history may be found in [17, 18, 21. In particular $\mathcal{C}^{s}\left(\mathbb{R}^{n}\right)$ is independent of the chosen resolution of unity according to $(2.3)$ (2.5) (equivalent norms). This justifies our omission of the subscript $\varphi$ in 2.6 in what 
follows. We recall the better known characterizations of the Hölder-Zygmund spaces $\mathcal{C}^{s}\left(\mathbb{R}^{n}\right)$ with $s>0$ in terms of differences. Let $C\left(\mathbb{R}^{n}\right)$ be the usual space of all complexvalued continuous bounded functions $f(x)$ in $\mathbb{R}^{n}$ normed by

$$
\left\|f\left|C\left(\mathbb{R}^{n}\right) \|=\sup _{x \in \mathbb{R}^{n}}\right| f(x) \mid .\right.
$$

Let

$$
\left(\Delta_{h}^{1} f\right)(x)=f(x+h)-f(x), \quad\left(\Delta_{h}^{l+1} f\right)(x)=\Delta_{h}^{1}\left(\Delta_{h}^{l} f\right)(x),
$$

where $x \in \mathbb{R}^{n}, h \in \mathbb{R}^{n}, l \in \mathbb{N}$, be the iterated differences in $\mathbb{R}^{n}$. Let $0<s<m \in \mathbb{N}$. Then $\mathcal{C}^{s}\left(\mathbb{R}^{n}\right)$ is the collection of all $f \in C\left(\mathbb{R}^{n}\right)$ such that

$$
\left\|\left.f\left|\mathcal{C}^{s}\left(\mathbb{R}^{n}\right) \|_{m}=\sup _{x \in \mathbb{R}^{n}}\right| f(x)|+\sup | h\right|^{-s}\left|\Delta_{h}^{m} f(x)\right|\right.
$$

is finite, where the second supremum is taken over all $x \in \mathbb{R}^{n}$ and $h \in \mathbb{R}^{n}$ with $0<|h| \leq 1$ (equivalent norms in $\mathcal{C}^{s}\left(\mathbb{R}^{n}\right)$ ).

We need a weighted extension of Definition 2.1

Definition 2.3. Let $s \in \mathbb{R}, \beta \in \mathbb{R}$ and

$$
w_{\beta}(x)=\left(1+|x|^{2}\right)^{\beta / 2}, \quad x \in \mathbb{R}^{n} .
$$

Then $\mathcal{C}^{s}\left(\mathbb{R}^{n}, \beta\right)$ is the collection of all $f \in S^{\prime}\left(\mathbb{R}^{n}\right)$ such that

$$
\left\|f\left|\mathcal{C}^{s}\left(\mathbb{R}^{n}, \beta\right)\|=\| w_{\beta} f\right| \mathcal{C}^{s}\left(\mathbb{R}^{n}\right)\right\|
$$

is finite.

This is the special case $\mathcal{C}^{s}\left(\mathbb{R}^{n}, \beta\right)=B_{\infty, \infty}^{s}\left(\mathbb{R}^{n}, \beta\right)$ of related spaces $A_{p, q}^{s}\left(\mathbb{R}^{n}, \beta\right)$, $A \in\{B, F\}, s \in \mathbb{R}, \beta \in \mathbb{R}$ and $0<p, q \leq \infty$. One reason for the distinguished role played by these weighted spaces is the following observation.

Proposition 2.4. Let $\varphi=\left\{\varphi_{j}\right\}_{j=0}^{\infty}$ be the resolution of unity according to 2.3 - 2.5. Let $s \in \mathbb{R}$ and $\beta \in \mathbb{R}$. Then $\mathcal{C}^{s}\left(\mathbb{R}^{n}, \beta\right)$ is the collection of all $f \in S^{\prime}\left(\mathbb{R}^{n}\right)$ such that

$$
\left\|f\left|\mathcal{C}^{s}\left(\mathbb{R}^{n}, \beta\right) \|_{\varphi}=\sup _{j \in \mathbb{N}_{0}, x \in \mathbb{R}^{n}} w_{\beta}(x) 2^{j s}\right|\left(\varphi_{j} \widehat{f}\right)^{\vee}(x) \mid\right.
$$

is finite (equivalent norms).

REMARK 2.5. This extension of 2.6 is a special case of the related assertion for the spaces $A_{p, q}^{s}\left(\mathbb{R}^{n}, \beta\right)$, having some history. The shortest available proof may be found $[3$, Section 4.2.2, pp. 156-158], based on [5].

We need a second crucial property of the spaces $\mathcal{C}^{s}\left(\mathbb{R}^{n}, \beta\right)$. A quasi-Banach space $A\left(\mathbb{R}^{n}\right)$ on $\mathbb{R}^{n}$ with

$$
S\left(\mathbb{R}^{n}\right) \hookrightarrow A\left(\mathbb{R}^{n}\right) \hookrightarrow S^{\prime}\left(\mathbb{R}^{n}\right), \quad A\left(\mathbb{R}^{n}\right) \subset L_{1}^{\text {loc }}\left(\mathbb{R}^{n}\right)
$$

(locally integrable functions in $\mathbb{R}^{n}$, whereas $\hookrightarrow$ stands for continuous embedding) is said to be a multiplication algebra if $f_{1} f_{2} \in A\left(\mathbb{R}^{n}\right)$ for $f_{1} \in A\left(\mathbb{R}^{n}\right), f_{2} \in A\left(\mathbb{R}^{n}\right)$ and if there is a constant $c^{\prime}>0$ such that

$$
\left\|f_{1} f_{2}\left|A\left(\mathbb{R}^{n}\right)\left\|\leq c^{\prime}\right\| f_{1}\right| A\left(\mathbb{R}^{n}\right)\right\| \cdot\left\|f_{2} \mid A\left(\mathbb{R}^{n}\right)\right\|
$$


for all $f_{1} \in A\left(\mathbb{R}^{n}\right), f_{2} \in A\left(\mathbb{R}^{n}\right)$. If $A\left(\mathbb{R}^{n}\right)$ is a multiplication algebra then

$$
A\left(\mathbb{R}^{n}\right) \hookrightarrow L_{\infty}\left(\mathbb{R}^{n}\right) .
$$

A short proof of this well known assertion (in the context of pointwise multipliers), further discussions and, in particular, related references, may be found in 29, Section 1.2 .6 , pp. 40-44].

Proposition 2.6. The space $\mathcal{C}^{s}\left(\mathbb{R}^{n}, \beta\right)$ according to Definition 2.3 is a multiplication algebra if and only if $s>0$ and $\beta \geq 0$.

Proof. Step 1. The space $\mathcal{C}^{0}\left(\mathbb{R}^{n}\right)$ is not a subset of $L_{1}^{\text {loc }}\left(\mathbb{R}^{n}\right)$. This follows from 20, Theorem 11.2, pp. 168-169] and the references given there, in particular to [14. It is a local property as a consequence of [20. p. 42, (5.13)] with a reference to [18, Theorem 2.4.7, p. 124]. This shows that $\mathcal{C}^{s}\left(\mathbb{R}^{n}, \beta\right)$ with $s \leq 0$ and $\beta \in \mathbb{R}$ is not a multiplication algebra. If $\beta<0$ then the unbounded function $w_{-\beta}$ belongs to $\mathcal{C}^{s}\left(\mathbb{R}^{n}, \beta\right)$. Then it follows from $(2.16)$ that $\mathcal{C}^{s}\left(\mathbb{R}^{n}, \beta\right)$ is not a multiplication algebra.

Step 2. Let $s>0$ and $\beta \geq 0$. From [25, Theorem 1.16, p. 12] it follows that $\mathcal{C}^{s}\left(\mathbb{R}^{n}\right)=$ $B_{\infty, \infty}^{s}\left(\mathbb{R}^{n}\right)$ is a multiplication algebra. Related references may be found in [25. Remark 1.17 , p. 12]. One may also consult [29, Section 1.4.2, pp. 77-83] for further information and wavelet arguments. Let $f \in \mathcal{C}^{s}\left(\mathbb{R}^{n}, \beta\right)$ and $g \in \mathcal{C}^{s}\left(\mathbb{R}^{n}, \beta\right)$. Then one obtains from 2.12 , 2.13) that

$$
\begin{aligned}
\left\|f g \mid \mathcal{C}^{s}\left(\mathbb{R}^{n}, \beta\right)\right\| & \leq c^{\prime}\left\|w_{\beta} f\left|\mathcal{C}^{s}\left(\mathbb{R}^{n}\right)\|\cdot\| g\right| \mathcal{C}^{s}\left(\mathbb{R}^{n}\right)\right\| \\
& \leq c^{\prime \prime}\left\|f\left|\mathcal{C}^{s}\left(\mathbb{R}^{n}, \beta\right)\|\cdot\| g\right| \mathcal{C}^{s}\left(\mathbb{R}^{n}, \beta\right)\right\| .
\end{aligned}
$$

This shows that $\mathcal{C}^{s}\left(\mathbb{R}^{n}, \beta\right)$ is a multiplication algebra.

We need a second preparation. Let $w \in S^{\prime}\left(\mathbb{R}^{n}\right)$. Then

$$
\begin{aligned}
W_{t} w(x)=\frac{1}{(4 \pi t)^{n / 2}} \int_{\mathbb{R}^{n}} \exp \left(-\frac{|x-y|^{2}}{4 t}\right) w(y) \mathrm{d} y & =\frac{1}{(4 \pi t)^{n / 2}}\left(w, \exp \left(-\frac{|x-\cdot|^{2}}{4 t}\right)\right), \quad t>0,
\end{aligned}
$$

$x \in \mathbb{R}^{n}$, is the well-known Gauss-Weierstrass semi-group which can be written on the Fourier side as

$$
\widehat{W_{t} w}(\xi)=e^{-t|\xi|^{2}} \widehat{w}(\xi), \quad \xi \in \mathbb{R}^{n}, t>0 .
$$

The Fourier transform is taken with respect to the space variables $x \in \mathbb{R}^{n}$. Of course, both 2.18 and 2.19 must be interpreted in the context of $S^{\prime}\left(\mathbb{R}^{n}\right)$. But we recall that 2.18 makes sense pointwise: It is the convolution of $w \in S^{\prime}\left(\mathbb{R}^{n}\right)$ and $g_{t}(y)=$ $(4 \pi t)^{-n / 2} e^{-|y|^{2} /(4 t)} \in S\left(\mathbb{R}^{n}\right)$. In particular,

$$
w * g_{t} \in C^{\infty}\left(\mathbb{R}^{n}\right), \quad\left|\left(w * g_{t}\right)(x)\right| \leq c_{t}^{\prime}\left(1+|x|^{2}\right)^{N / 2}, \quad x \in \mathbb{R}^{n},
$$

for some $c_{t}^{\prime}>0$ and some $N \in \mathbb{N}$. Further explanations and related references may be found in [26, Section 4.1]. If $w$ and $f$ are regular distributions, subject to some restrictions, 
then

$$
\begin{aligned}
& W(x, t) \\
& =\frac{1}{(4 \pi t)^{n / 2}} \int_{\mathbb{R}^{n}} \exp \left(-\frac{|x-y|^{2}}{4 t}\right) w(y) \mathrm{d} y+\frac{1}{(4 \pi)^{n / 2}} \int_{0}^{t} \int_{\mathbb{R}^{n}} \frac{\exp \left(-\frac{|x-y|^{2}}{4(t-\tau)}\right)}{(t-\tau)^{n / 2}} f(y, \tau) \mathrm{d} y \mathrm{~d} \tau \\
& =W_{t} w(x)+\left(\int_{0}^{t} W_{t-\tau} f_{\tau} \mathrm{d} \tau\right)(x)
\end{aligned}
$$

$x \in \mathbb{R}^{n}, t>0$, with $f_{\tau}(y)=f(y, \tau)$ is the well-known unique solution of the classical Cauchy problem

$$
\begin{aligned}
\partial_{t} W(x, t)-\Delta W(x, t) & =f(x, t), & & x \in \mathbb{R}^{n}, \quad t>0, \\
W(x, 0) & =w(x), & & x \in \mathbb{R}^{n} .
\end{aligned}
$$

By using 2.19, equality 2.21 can be written on the Fourier side in terms of the Duhamel formula

$$
\widehat{W}(\xi, t)=e^{-t|\xi|^{2}} \widehat{w}(\xi)+\int_{0}^{t} e^{-(t-\tau)|\xi|^{2}} \widehat{f}_{\tau}(\xi) \mathrm{d} \tau,
$$

$\xi \in \mathbb{R}^{n}, t>0$. Details and references may be found in [19, Sections 3.3.4-3.3.6, pp. 169172], [16. Section 2.5.2, pp. 190-192] and [25, p. 160]. Let $X$ be a suitable space, say, of type $A_{p, q}^{s}\left(\mathbb{R}^{n}\right)$ and let $T>0, b \in \mathbb{R}$ and $1 \leq v \leq \infty$. Then the Banach spaces $L_{v}((0, T), b, X)$ normed by

$$
\left\|f \mid L_{v}((0, T), b, X)\right\|=\left(\int_{0}^{T} t^{b v}\|f(\cdot, t) \mid X\|^{v} \mathrm{~d} t\right)^{1 / v},
$$

with the usual modification if $v=\infty$, are the basic spaces of our approach to the NavierStokes equations in [25, 26] and to the Keller-Segel equations of chemotaxis in [28]. For this purpose we converted the related equations with the help of 2.21 into fixed point problems in spaces of this type. Now we are doing the same with respect to the Lengyel-Epstein equations (1.1), (1.2). The peculiar nature of these equations requires to specify the spaces in 2.25 . Let $X=\mathcal{C}^{s}\left(\mathbb{R}^{n}, \beta\right)$ be the spaces introduced in Definition 2.3 and covered by Propositions 2.4 and 2.6 Then $C\left((0, T), \mathcal{C}^{s}\left(\mathbb{R}^{n}, \beta\right)\right)$ is the usual Banach space of bounded continuous functions $u(\cdot, t)$ belonging for any $t$ with $0<t<T$ to $\mathcal{C}^{s}\left(\mathbb{R}^{n}, \beta\right)$ and normed by

$$
\left\|u\left|C\left((0, T), \mathcal{C}^{s}\left(\mathbb{R}^{n}, \beta\right)\right)\left\|=\sup _{0<t<T}\right\| u(\cdot, t)\right| \mathcal{C}^{s}\left(\mathbb{R}^{n}, \beta\right)\right\| .
$$

Any element $u(\cdot, t)$ of this space can be extended to $t \geq T$ and to $t \leq 0$ by zero. Then the resulting Banach space may be considered as a subspace of $S^{\prime}\left(\mathbb{R}^{n+1}\right)$.

2.2. Basic assertions. Let

$$
\begin{aligned}
\partial_{t} u-\sigma_{u} \Delta u & =a-u-\frac{4 u v}{1+u^{2}}, & & x \in \mathbb{R}^{n}, 0<t<T, \\
\partial_{t} v-\sigma_{v} \Delta v & =b c u-\frac{c u v}{1+u^{2}}, & x & \in \mathbb{R}^{n}, 0<t<T, \\
u(\cdot, 0) & =u_{0}, & x & \in \mathbb{R}^{n}, \\
v(\cdot, 0) & =v_{0}, & x & \in \mathbb{R}^{n},
\end{aligned}
$$


be the Lengyel-Epstein system (1.1), 1.2$)$ in $\mathbb{R}^{n} \times(0, T)$, complemented by the initial data $u_{0}(x), v_{0}(x)$. As before $\partial_{t}=\partial / \partial t$ whereas $\Delta=\sum_{j=1}^{n} \partial_{j}^{2}=\sum_{j=1}^{n} \partial^{2} / \partial x_{j}^{2}$ is the Laplacian with respect to the space variables $x=\left(x_{1}, \ldots, x_{n}\right) \in \mathbb{R}^{n}$. Let again $\sigma_{u}>0$ and $\sigma_{v}>0$ be the diffusion coefficients. We deal with these equations in the context of the above spaces $\mathcal{C}^{s}\left(\mathbb{R}^{n}, \beta\right), s>0, \beta \geq 0$, according to Definition 2.3 . This requires that in addition to the constants $b \in \mathbb{C}$ and $c \in \mathbb{C}$ the constant $a$ in 1.1 must be adapted by

$$
a(x)=c_{a}\left(1+|x|^{2}\right)^{-\beta / 2}=c_{a} w_{\beta}(x)^{-1}, \quad c_{a} \in \mathbb{C} .
$$

As already indicated we convert the Cauchy problem $(2.27)-(2.30)$ into a fixed point problem and abbreviate for this purpose the related right-hand sides of $(2.27), 22.28$ by

$$
f(u, v)=a-u-\frac{4 u v}{1+u^{2}} \quad \text { and } \quad g(u, v)=b c u-\frac{c u v}{1+u^{2}} .
$$

Modifying (2.22), 2.23) by 2.27) -2.30 one has to replace (2.21) by

$$
\begin{aligned}
& \left(U_{u_{0}, v_{0}}(u, v)\right)(x, t) \\
& \quad=W_{\sigma_{u} t} u_{0}(x)+\left[\int_{0}^{\sigma_{u} t} \sigma_{u}^{-1} W_{\sigma_{u} t-\tau} f\left(u\left(\cdot, \sigma_{u}^{-1} \tau\right), v\left(\cdot, \sigma_{u}^{-1} \tau\right)\right) \mathrm{d} \tau\right](x), \\
& \left(V_{u_{0}, v_{0}}(u, v)\right)(x, t) \\
& \quad=W_{\sigma_{v} t} v_{0}(x)+\left[\int_{0}^{\sigma_{v} t} \sigma_{v}^{-1} W_{\sigma_{v} t-\tau} g\left(u\left(\cdot, \sigma_{v}^{-1} \tau\right), v\left(\cdot, \sigma_{v}^{-1} \tau\right)\right) \mathrm{d} \tau\right](x) .
\end{aligned}
$$

But this modification is a technical matter and can be neglected in the context of our qualitative arguments below based on $\mathcal{C}^{s}\left(\mathbb{R}^{n}, \beta\right)$ according to Definition 2.3 and Proposition 2.6. In addition to $C\left((0, T), \mathcal{C}^{s}\left(\mathbb{R}^{n}, \beta\right)\right)$ in 2.26 we introduce the space $X^{s, \beta}(T)$ consisting of all pairs $u, v \in C\left((0, T), \mathcal{C}^{s}\left(\mathbb{R}^{n}, \beta\right)\right)$ and normed by

$$
\left\|(u, v) \mid X^{s, \beta}(T)\right\|=\max \left(\left\|u\left|C\left((0, T), \mathcal{C}^{s}\left(\mathbb{R}^{n}, \beta\right)\right)\|,\| v\right| C\left((0, T), \mathcal{C}^{s}\left(\mathbb{R}^{n}, \beta\right)\right)\right\|\right) .
$$

Let

$$
X^{s, \beta}(T)_{\delta}=\left\{(u, v) \in X^{s, \beta}(T):\left\|(u, v) \mid X^{s, \beta}(T)\right\| \leq \delta\right\}
$$

be the $\delta$-ball in this space, $\delta>0$. By the same arguments as in [28, p. 80] we may assume that $\mathcal{C}^{s}\left(\mathbb{R}^{n}, \beta\right)$ with $s>0$ and $\beta \geq 0$ as in Proposition 2.6 is normed such that

$$
\left\|f g\left|\mathcal{C}^{s}\left(\mathbb{R}^{n}, \beta\right)\|\leq\| f\right| \mathcal{C}^{s}\left(\mathbb{R}^{n}, \beta\right)\right\| \cdot\left\|g \mid \mathcal{C}^{s}\left(\mathbb{R}^{n}, \beta\right)\right\|, \quad f, g \in \mathcal{C}^{s}\left(\mathbb{R}^{n}, \beta\right),
$$

and

$$
|f(x)| \leq\left\|f \mid \mathcal{C}^{s}\left(\mathbb{R}^{n}, \beta\right)\right\|, \quad f \in \mathcal{C}^{s}\left(\mathbb{R}^{n}, \beta\right), \quad x \in \mathbb{R}^{n} .
$$

Theorem 2.7. Let $s>0$ and $\beta \geq 0$. Let $b, c$ and $c_{a}$ in 2.31 be complex numbers. Then there are positive numbers $\varepsilon, \delta$ and $T$ such that 2.27) 2.30 with $u_{0} \in \mathcal{C}^{s}\left(\mathbb{R}^{n}, \beta\right)$, $v_{0} \in \mathcal{C}^{s}\left(\mathbb{R}^{n}, \beta\right)$ and

$$
\left\|u_{0}\left|\mathcal{C}^{s}\left(\mathbb{R}^{n}, \beta\right)\|\leq \varepsilon, \quad\| v_{0}\right| \mathcal{C}^{s}\left(\mathbb{R}^{n}, \beta\right)\right\| \leq \varepsilon
$$

has a unique solution $(u, v)$ in $X^{s, \beta}(T)_{\delta}$. 
Proof. We apply Banach's contraction theorem to 2.33 , 2.34 with $\sigma_{u}=\sigma_{v}=1$ for simplicity. Since the Gauss-Weierstrass kernels in (2.18) are positive one obtains

$$
\begin{aligned}
& \left\|U_{u_{0}, v_{0}}(u, v) \mid C\left((0, T), \mathcal{C}^{s}\left(\mathbb{R}^{n}, \beta\right)\right)\right\| \\
& \quad \leq c^{\prime}\left\|u_{0}\left|\mathcal{C}^{s}\left(\mathbb{R}^{n}, \beta\right)\left\|+c^{\prime} T\right\| f(u, v)\right| C\left((0, T), \mathcal{C}^{s}\left(\mathbb{R}^{n}, \beta\right)\right)\right\|
\end{aligned}
$$

for some constant $c^{\prime}>0$ and a counterpart for $V_{u_{0}, v_{0}}(u, v)$. We add a technical comment about this estimate in Remark 2.8 below. What follows is justified if $\delta>0$ in 2.36 is sufficiently small. In particular, if $(u, v) \in X^{s, \beta}(T)_{\delta}$ then we may assume that $\left|u^{2}(x, t)\right|<1 / 2$ uniformly in $x \in \mathbb{R}^{n}$ and $0<t<T$. Then it follows from 2.37), 2.38) that

$$
\left|\frac{1}{1-q}\right|=\left|\sum_{l=0}^{\infty} q^{l}\right|<2 \quad \text { where } \quad q=q(x, t)=-u^{2}(x, t)
$$

uniformly in $x \in \mathbb{R}^{n}$ and $0<t<T$ as needed in 2.32, 2.33. Again at the expense of $\delta>0$ small one has

$$
\left\|(1-q(x, t))^{-1} \mid C\left((0, T), \mathcal{C}^{s}\left(\mathbb{R}^{n}, \beta\right)\right)\right\|<2 .
$$

Inserted in 2.32 and 2.40 one has $f(u, v) \in C\left((0, T), \mathcal{C}^{s}\left(\mathbb{R}^{n}, \beta\right)\right)$ and for some $c^{\prime}>0$, $c^{\prime \prime}>0$

$$
\left\|U_{u_{0}, v_{0}}(u, v) \mid C\left((0, T), \mathcal{C}^{s}\left(\mathbb{R}^{n}, \beta\right)\right)\right\| \leq c^{\prime} \varepsilon+c^{\prime \prime} T<\delta,
$$

if $\varepsilon>0$ in 2.39) and $T>0$ are chosen sufficiently small. Similarly for $V_{u_{0}, v_{0}}(u, v)$. In particular $\left(U_{u_{0}, v_{0}}, V_{u_{0}, v_{0}}\right)$ maps $X^{s, \beta}(T)_{\delta}$ into itself. We prove that this map is a contraction. Let $\left(u_{1}, v_{1}\right) \in X^{s, \beta}\left(\mathbb{R}^{n}\right)_{\delta}$ and $\left(u_{2}, v_{2}\right) \in X^{s, \beta}\left(\mathbb{R}^{n}\right)_{\delta}$. Then it follows from 2.33 (again with $\sigma_{u}=1$ ) that

$$
\begin{aligned}
& \left(U_{u_{0}, v_{0}}\left(u_{1}, v_{1}\right)-U_{u_{0}, v_{0}}\left(u_{2}, v_{2}\right)\right)(x, t) \\
& \quad=\left(\int_{0}^{t} W_{t-\tau}\left[f\left(u_{1}(\cdot, \tau), v_{1}(\cdot, \tau)\right)-f\left(u_{2}(\cdot, \tau), v_{2}(\cdot, \tau)\right)\right] \mathrm{d} \tau\right)(x) .
\end{aligned}
$$

By 2.32 and $u_{2}=u_{1}+\left(u_{2}-u_{1}\right), v_{2}=v_{1}+\left(v_{2}-v_{1}\right)$ we have

$$
\begin{aligned}
f\left(u_{1}, v_{1}\right)-f\left(u_{2}, v_{2}\right) & =u_{2}-u_{1}+4 \frac{\left(1+u_{1}^{2}\right) u_{2} v_{2}-\left(1+u_{2}^{2}\right) u_{1} v_{1}}{\left(1+u_{1}^{2}\right)\left(1+u_{2}^{2}\right)} \\
& =\frac{\left(u_{1}-u_{2}\right) P_{1}+\left(v_{1}-v_{2}\right) P_{2}}{\left(1+u_{1}^{2}\right)\left(1+u_{2}^{2}\right)}
\end{aligned}
$$

where $P_{1}, P_{2}$ are polynomials in $u_{1}, u_{2}, v_{1}, v_{2}$ of fourth degree. Inserted in 2.44) one can argue as above resulting in

$$
\begin{aligned}
\| U_{u_{0}, v_{0}}\left(u_{1}, v_{1}\right)-U_{u_{0}, v_{0}}\left(u_{2}, v_{2}\right) & \mid C\left((0, T), \mathcal{C}^{s}\left(\mathbb{R}^{n}, \beta\right)\right) \| \\
& \leq \lambda\left\|\left(u_{1}, v_{1}\right)-\left(u_{2}, v_{2}\right) \mid X^{s, \beta}(T)\right\|
\end{aligned}
$$

for some $0<\lambda<1$ at the expense of a small $T>0$. Similarly for $V_{u_{0}, v_{0}}\left(u_{1}, v_{1}\right)-$ $V_{u_{0}, v_{0}}\left(u_{2}, v_{2}\right)$. Then $\left(U_{u_{0}, v_{0}}, V_{u_{0}, v_{0}}\right)$ is a contraction in $X^{s, \beta}\left(\mathbb{R}^{n}\right)_{\delta}$. The assertions of the theorem follow from Banach's contraction theorem. 
REMARK 2.8. With $y=x+\sqrt{t} z$ one can rewrite 2.18 by

$$
W_{t} w(x)=\frac{1}{(4 \pi)^{n / 2}} \int_{\mathbb{R}^{n}} e^{-|z|^{2} / 4} w(x+\sqrt{t} z) \mathrm{d} z, \quad t>0, \quad x \in \mathbb{R}^{n} .
$$

Then

$$
\left\|W_{t} w\left|\mathcal{C}^{s}\left(\mathbb{R}^{n}\right)\|\leq\| w\right| \mathcal{C}^{s}\left(\mathbb{R}^{n}\right)\right\|, \quad t>0,
$$

follows from the translation invariance of $\mathcal{C}^{s}\left(\mathbb{R}^{n}\right)=\mathcal{C}^{s}\left(\mathbb{R}^{n}, 0\right)$. If $\beta>0$ then one can rely on $(2.12$ and

$$
w_{\beta}(x) \leq c^{\prime}\left(1+\sqrt{t}|z|^{2}\right)^{\beta / 2} w_{\beta}(x+\sqrt{t} z)
$$

for some $c^{\prime}>0$ resulting in

$$
\left\|W_{t} w\left|\mathcal{C}^{s}\left(\mathbb{R}^{n}, \beta\right)\left\|\leq c^{\prime \prime}\right\| w\right| \mathcal{C}^{s}\left(\mathbb{R}^{n}, \beta\right)\right\|, \quad 0<t<T,
$$

for some $c^{\prime \prime}>0$. This justifies 2.40 .

REMARK 2.9. If $\beta=0$ then $a(x)$ in 2.31 is a constant and 2.27 2.30 coincides with (1.1), 1.2 complemented by suitable initial data. Usually (1.1)-1.3 is considered in bounded (smooth) domains $\Omega$ in $\mathbb{R}^{n}$ where related (zero) Dirichlet or Neumann boundary data at $\partial \Omega$ play only a marginal role. The incorporation of the weight $w_{\beta}$ according to (2.11) makes sense from the point of view of applications. If $u_{0}, v_{0}$ in $(2.29), 2.30$ have compact support and if the constant $a$ in $(1.1)$ is adapted according to (2.31) then the solutions $u(x, t), v(x, t)$ belonging to $\mathcal{C}^{s}\left(\mathbb{R}^{n}, \beta\right)$ decay rapidly. This may be considered as a reasonable substitute for dealing with $11.1,01.2$ in bounded domains $\Omega$ in $\mathbb{R}^{n}$. We discuss in Section 2.3 below some more specific properties of the Lengyel-Epstein equations nearer to the chemical and biological origin.

Solutions of 2.27 - 2.30 originating from fixed point theorems are called mild. Furthermore one may ask of whether these solutions are also strong, what means in the above context

$$
\begin{array}{ll}
u(\cdot, t) \rightarrow u_{0}(\cdot) & \text { in } \mathcal{C}^{s}\left(\mathbb{R}^{n}, \beta\right) \text { if } t \rightarrow 0, \\
v(\cdot, t) \rightarrow v_{0}(\cdot) & \text { in } \mathcal{C}^{s}\left(\mathbb{R}^{n}, \beta\right) \text { if } t \rightarrow 0,
\end{array}
$$

what can also be written as

$$
u \in C\left([0, T), \mathcal{C}^{s}\left(\mathbb{R}^{n}, \beta\right)\right), \quad v \in C\left([0, T), \mathcal{C}^{s}\left(\mathbb{R}^{n}, \beta\right)\right)
$$

incorporating continuity at $t=0$. Let $\stackrel{\circ}{\mathcal{C}}\left(\mathbb{R}^{n}, \beta\right)$ be the completion of $S\left(\mathbb{R}^{n}\right)$ in $\mathcal{C}^{s}\left(\mathbb{R}^{n}, \beta\right)$.

COROLlary 2.10. Under the additional assumption

$$
u_{0} \in \stackrel{\circ}{\mathcal{C}}^{s}\left(\mathbb{R}^{n}, \beta\right), \quad v_{0} \in \stackrel{\circ}{\mathcal{C}}^{s}\left(\mathbb{R}^{n}, \beta\right)
$$

the solutions $u$ and $v$ in Theorem 2.7 are mild and strong.

Proof. By 2.33), 2.34 (again with $\sigma_{u}=\sigma_{v}=1$ ) and the arguments resulting in 2.40) one has

$$
\begin{array}{ll}
\left\|u(\cdot, t)-W_{t} u_{0}(\cdot) \mid \mathcal{C}^{s}\left(\mathbb{R}^{n}, \beta\right)\right\| \leq c^{\prime} t, & 0<t<T, \\
\left\|v(\cdot, t)-W_{t} v_{0}(\cdot) \mid \mathcal{C}^{s}\left(\mathbb{R}^{n}, \beta\right)\right\| \leq c^{\prime} t, & 0<t<T .
\end{array}
$$

Using (2.50) one can argue as in [28, pp. 42-43] with a reference to [26, pp. 121-122] as far as initial data $u_{0} \in S\left(\mathbb{R}^{n}\right), v_{0} \in S\left(\mathbb{R}^{n}\right)$ are concerned. 
2.3. Further properties. So far we ignored that the coefficients $a, b, c$ in (1.1), (1.2) are positive and that we are interested in positive solutions $u(x, t), v(x, t)$ under the assumption that the underlying initial data $u_{0}(x), v_{0}(x)$ are positive. We switch again from bounded domains $\Omega$ to $\mathbb{R}^{n}$ compensated by the requested strong decay of the initial data $u_{0}, v_{0}$ and the solutions $u, v$ with respect to the space variables. Then $a>0$ is replaced by $c_{a}>0$ in 2.31). Theorem 2.7 can now be complemented as follows. We say that $(u, v) \in X^{s, \beta}(T)_{\delta}$ is positive if $u(x, t)>0$ and $v(x, t)>0$ for all $x \in \mathbb{R}^{n}$ and $0<t<T$.

Proposition 2.11. Let $s>0$ and $\beta \geq 0$. Let $b, c$ and $c_{a}$ in $(2.31$ be positive numbers. Then there are positive numbers $\varepsilon, \delta$ and $T$ such that 2.27$)-(2.30)$ with $u_{0} \in \mathcal{C}^{s}\left(\mathbb{R}^{n}, \beta\right)$, $v_{0} \in \mathcal{C}^{s}\left(\mathbb{R}^{n}, \beta\right), u_{0}(x) \geq 0, v_{0}(x) \geq 0$ if $x \in \mathbb{R}^{n}$ and with 2.39 has a unique positive solution $(u, v)$ in $X^{s, \beta}(T)_{\delta}$.

Proof. We prove that under the indicated conditions the unique solution according to Theorem 2.7 is positive if the positive numbers $\varepsilon, \delta, T$ are sufficiently small. With $w=a$ according to 2.31 in 2.47), using in addition a suitable counterpart of 2.49), one has

$$
\left(1+|x|^{2}\right)^{\beta / 2}\left(W_{t} a\right)(x)=c_{a}(4 \pi)^{-n / 2} \int_{\mathbb{R}^{n}} e^{-|z|^{2} / 4} \frac{\left(1+|x|^{2}\right)^{\beta / 2}}{\left(1+|x+\sqrt{t} z|^{2}\right)^{\beta / 2}} \mathrm{~d} z \geq C_{a}>0
$$

independently of $x \in \mathbb{R}^{n}$ and $t$ with $0<t<T$. Then one obtains

$$
\left(\int_{0}^{t} W_{t-\tau} a \mathrm{~d} \tau\right)(x) \geq C_{a} \frac{t}{\left(1+|x|^{2}\right)^{\beta / 2}}, \quad x \in \mathbb{R}^{n}, \quad 0<t<T .
$$

The usual proof of Banach's contraction theorem is based on the iterations

$$
\begin{aligned}
u^{j+1}(x, t) & =W_{t} u_{0}(x)+\left(\int_{0}^{t} W_{t-\tau} f\left(u^{j}(\cdot, \tau), v^{j}(\cdot, \tau)\right) \mathrm{d} \tau\right)(x), \\
v^{j+1}(x, t) & =W_{t} v_{0}(x)+\left(\int_{0}^{t} W_{t-\tau} g\left(u^{j}(\cdot, \tau), v^{j}(\cdot, \tau)\right) \mathrm{d} \tau\right)(x),
\end{aligned}
$$

$j \in \mathbb{N}$, with $f, g$ as in 2.31, 2.32 and 2.33, 2.34 assuming again $\sigma_{u}=\sigma_{v}=1$. One may begin with $u^{1}(x, t)=v^{1}(x, t)=0$. Then $u^{2}(x, t)>0, v^{2}(x, t) \geq 0$ and the related norms according to the above procedure and (2.10), 2.12 are small. Iteration, (2.57), (2.58) as the dominant terms in 2.32, 2.33 with

$$
g(u, v) \sim b c u\left(1-\frac{v}{b\left(1+u^{2}\right)}\right) \sim b c u
$$

show that $u^{j}(x, t)>0, v^{j}(x, t)>0,3 \leq j \in \mathbb{N}$, always under the assumption that $\varepsilon, \delta$ and $T$ are sufficiently small positive numbers.

REMARK 2.12. The above arguments and the underlying fixed norm according to 2.10 show that

$$
\begin{aligned}
& 0 \leq W_{t} u_{0}(x) \leq u(x, t) \leq \frac{\delta}{\left(1+|x|^{2}\right)^{\beta / 2}}, \\
& 0 \leq W_{t} v_{0}(x) \leq v(x, t) \leq \frac{\delta}{\left(1+|x|^{2}\right)^{\beta / 2}},
\end{aligned}
$$

$x \in \mathbb{R}^{n}, 0<t<T$. This will be of some service for us. 
As mentioned above the strong decay of $u(x, t), v(x, t)$ as indicated in 2.62 may be considered as a substitute for dealing with Lengyel-Epstein systems in bounded (smooth) domains. This applies also to the following observation.

Corollary 2.13. Let $s>0$ and $\beta>n \in \mathbb{N}$. Let $b, c$ and $c_{a}$ in (2.31) be positive numbers. Let $u(x, t), v(x, t)$ be the unique positive solution of 2.27) 2.30 according to Proposition 2.11. Then for $0<t<T$,

$$
\begin{aligned}
0 & \leq \int_{\mathbb{R}^{n}} u(x, t) \mathrm{d} x-\int_{\mathbb{R}^{n}} u_{0}(x) \mathrm{d} x \leq t \int_{\mathbb{R}^{n}} a(x) \mathrm{d} x, \\
0 & \leq \int_{\mathbb{R}^{n}} v(x, t) \mathrm{d} x-\int_{\mathbb{R}^{n}} v_{0}(x) \mathrm{d} x \leq b c t \int_{\mathbb{R}^{n}} u_{0}(x) \mathrm{d} x+\frac{b c}{2} t^{2} \int_{\mathbb{R}^{n}} a(x) \mathrm{d} x .
\end{aligned}
$$

Proof. The assumption $\beta>n$ in 2.12, 2.10 and 2.35, 2.36 ensures that the initial data $u_{0}, v_{0}$, the function $a$ in 2.31 and the positive solutions $u(x, t), v(x, t)$ are integrable with respect to $x \in \mathbb{R}^{n}$. The left-hand sides of (2.63), 2.64) follow from $(2.62)$ and

$$
\int_{\mathbb{R}^{n}} W_{t} u_{0}(x) \mathrm{d} x=\int_{\mathbb{R}^{n}} u_{0}(x) \mathrm{d} x, \quad \int_{\mathbb{R}^{n}} W_{t} v_{0}(x) \mathrm{d} x=\int_{\mathbb{R}^{n}} v_{0}(x) \mathrm{d} x .
$$

We rely on the identities 2.33), 2.34) with $u(x, t), v(x, t)$ on the related left-hand sides and $f, g$ as in (2.32). Then the right-hand side of 2.63 follows by integration and the above reasoning in the proof of Proposition 2.11 and Remark 2.12. In the corresponding integration of (2.34) with $v(x, t)$ on the left-hand side we use (2.63). This proves, again on the basis of the above reasoning, the right-hand side of (2.64).

REMARK 2.14. In connection with the so-called Keller-Segel equations of chemotaxis one asks for the conservation property

$$
\int_{\mathbb{R}^{n}} u(x, t) \mathrm{d} x=\int_{\mathbb{R}^{n}} u_{0}(x) \mathrm{d} x, \quad 0<t<T,
$$

for the total mass of cells, where $u(x, t)$ is the cell density and $u_{0}(x)$ the related initial distribution. We refer the reader to [28, Section 4.9, pp. 60-61]. For the positive solutions $u(x, t)$ and $v(x, t)$ of $(1.1),(1.2)$ according to Proposition 2.11 and Corollary 2.13 one may ask the same questions, although the situation is different because of the chemical reactions. This applies both to the involved non-linearities and the acting (competing) chemicals $u(x, t)$ and $v(x, t)$. Nevertheless the outcome 2.63), 2.64 looks reasonable. Both

$$
\int_{\mathbb{R}^{n}} u(x, t) \mathrm{d} x \text { and } \int_{\mathbb{R}^{n}} v(x, t) \mathrm{d} x
$$

grow at most linearly in time as long as Corollary 2.13 can be applied. Furthermore, the functions in 2.67) are monotonically increasing in time. This can be seen if one shifts the Cauchy problem from $t=0$ to an admitted $t_{0}>0$ and uses that the related solutions are unique. In addition, $u(x, t)$ and $v(x, t)$ are $C^{\infty}$ functions in $\mathbb{R}^{n} \times(0, T)$.

\section{Turing patterns and Faber splines}

3.1. The hypothesis: Let Turing patterns be. We described in Section 1 Turing patterns in connection with the Lengyel-Epstein system (1.1), 1.2) usually considered in bounded domains $\Omega$ in $\mathbb{R}^{n}$. After some general assertions for the equations 2.27)-2.30 in Section 2.2 we dealt in Section 2.3 with a few more specific properties nearer to the original 
task. In particular instead of function spaces in bounded domains we relied on the spaces $\mathcal{C}^{s}\left(\mathbb{R}^{n}, \beta\right)$ in Definition 2.3 and $2.10,2.13$ ensuring a rapid decay if $\beta$ is large of possible solutions of 2.27 -2.30 for suitable initial data, say, with compact support. Combined with the specific properties derived in Section 2.3 and the experimental observations as described in Section 1 we convert the question

\section{how large Turing patterns must be}

into the study of the Hypothesis below. We will rely on one-dimensional Faber splines (in good agreement with observed Turing patterns). First we describe our set-up.

Let the unit square $Q=(0,1)^{2}$ in the plane $\mathbb{R}^{2}$ be the underlying domain. We imitate the vertical patterns as described in Section 1 by

$$
S_{J, m}=\left\{\left(x_{1}, x_{2}\right) \in Q: 2^{-J} m<x_{1}<2^{-J}(m+1), 0<x_{2}<1\right\},
$$

$J \in \mathbb{N} ; m=0, \ldots, 2^{J}-1$. Let

$$
\partial S^{J}=\bigcup_{m=0}^{2^{J}-1} \partial S_{J, m}=\bigcup_{m=0}^{2^{J}}\left\{x=\left(x_{1}, x_{2}\right) \in \bar{Q}: x_{1}=2^{-J} m, 0 \leq x_{2} \leq 1\right\}
$$

be the collection of the boundaries of the stripes in 3.1 . Let $\mathcal{C}^{s}\left(\mathbb{R}^{2}\right)$ with $s>0$ be the Hölder-Zygmund spaces as introduced in Definition 2.1 and Remark 2.2 Let

$$
\widetilde{\mathcal{C}}^{s}(Q)=\left\{f \in \mathcal{C}^{s}\left(\mathbb{R}^{2}\right): \operatorname{supp} f \subset \bar{Q}\right\}, \quad s>0,
$$

where $\bar{Q}=[0,1]^{2}$. Similarly

$$
\widetilde{\mathcal{C}}^{s}(I)=\left\{f \in \mathcal{C}^{s}(\mathbb{R}): \operatorname{supp} f \subset \bar{I}\right\}, \quad s>0,
$$

where $I=(0,1)$ and $\bar{I}=[0,1]$. There is a constant $c^{\prime}>0$ such that

$$
\left\|u\left(\cdot, x_{2}\right)\left|\mathcal{C}^{s}(\mathbb{R})\left\|\leq c^{\prime}\right\| u\right| \mathcal{C}^{s}\left(\mathbb{R}^{2}\right)\right\|, \quad u \in \widetilde{\mathcal{C}}^{s}(Q),
$$

uniformly for all $0 \leq x_{2} \leq 1$. This follows from the trace theorem

$$
\operatorname{tr}_{x_{2}}: \quad \widetilde{\mathcal{C}}^{s}(Q) \hookrightarrow \widetilde{\mathcal{C}}^{s}(I) \quad \text { with } \quad \operatorname{tr}_{x_{2}} u=u\left(\cdot, x_{2}\right)
$$

which is a special case of [18, Corollary 4.4.2, p. 219].

Hypothesis 3.1. Let $s>0$ and

$$
u_{0} \in \widetilde{\mathcal{C}}^{s}(Q), \quad v_{0} \in \widetilde{\mathcal{C}}^{s}(Q), \quad u_{0} \geq 0, \quad v_{0} \geq 0 .
$$

Let $T>0$ and

$$
u(\cdot, T) \in \widetilde{\mathcal{C}}^{s}(Q), \quad v(\cdot, T) \in \widetilde{\mathcal{C}}^{s}(Q), \quad u \geq 0, \quad v \geq 0
$$

such that

$$
\begin{aligned}
& 0<\int_{Q} u_{0}(x) \mathrm{d} x \leq \int_{Q} u(x, T) \mathrm{d} x \leq \int_{Q} u_{0}(x) \mathrm{d} x+T \\
& 0<\int_{Q} v_{0}(x) \mathrm{d} x \leq \int_{Q} v(x, T) \mathrm{d} x \leq \int_{Q} v_{0}(x) \mathrm{d} x+T,
\end{aligned}
$$

and

$$
\begin{gathered}
\left\|u(\cdot, T)\left|\mathcal{C}^{s}(Q)\|\leq\| u_{0}\right| \mathcal{C}^{s}(Q)\right\|+T \\
\left\|v(\cdot, T)\left|\mathcal{C}^{s}(Q)\|\leq\| v_{0}\right| \mathcal{C}^{s}(Q)\right\|+T
\end{gathered}
$$


Let furthermore

$$
u(x, T)=v(x, T)=0 \quad \text { if } \quad x \in \partial S^{J}, \quad J \in \mathbb{N},
$$

according to 3.2 .

REMARK 3.2. We interpret $u_{0}, v_{0}$ according to (3.7) with (3.3) as the initial data in Theorem 2.7. Corollary 2.10 and Proposition 2.11. Corollary 2.13 In particular, (3.8) may be considered as a reasonable adaption of $u(\cdot, t) \in \mathcal{C}^{s}\left(\mathbb{R}^{2}, \beta\right), v(\cdot, t) \in \mathcal{C}^{s}\left(\mathbb{R}^{2}, \beta\right)$ in Corollary 2.13 In other words, (3.7)-3.12 reflect faithfully crucial properties of the Lengyel-Epstein equations as considered above. The additional decisive request (3.13) can be rephrased as

\section{Let Turing patterns be}

adapting the well-known epitaph of Alexander Pope (1688-1744)

Nature, and Nature's Laws lay hid in Night.

God said, Let Newton be! and All was Light.

This again is in a good agreement with observed periodic (in space) vertical Turing patterns as described in [2, 6, 7]. It might also be considered as a complement to the experimental data, numerical simulations and analytical justifications as described in the books and papers mentioned in Section 1. It is our main aim to contribute to the question

How large Turing patterns must be?

3.2. Faber splines. We rely on Faber splines in one dimension as considered in [24, Section 3.4.1, pp. 58-62], based on [23, Sections 3.5.2, 3.5.3, pp. 167-173]. The basic Faber spline $V_{l}(x), l \in \mathbb{N}_{0}, x \in \mathbb{R}$ has the following properties:

(i) The function $V_{l}$ has classical derivatives up to order $2 l$ in $\mathbb{R}$.

(ii) The restriction of $V_{l}$ to each interval $\left(m, m+\frac{1}{2}\right)$ with $2 m \in \mathbb{Z}$ is a polynomial of order $2 l+1$.

(iii) There are constants $c^{\prime}>0, \nu>0$, such that

$$
\left|V_{l}(x)\right| \leq c^{\prime} 2^{-2 \nu|x|}, \quad x \in \mathbb{R} .
$$

In contrast to the classical compactly supported hat function $V_{0}$ we have now only the exponential decay (3.14) for $V_{l}, l \in \mathbb{N}$, with some $\nu=\nu_{l}>0$. According to [24, Definition 3.9, p. 59] $=$ [23. Definition 3.38, p. 169]

$$
\left\{v_{j, m}^{l}(x)=V_{l}\left(2^{j} x-m\right): j \in \mathbb{N}_{0}, m \in \mathbb{Z}\right\}
$$

is called a Faber spline system of order $l$. We give now a brief description of expansions in terms of Faber splines specifying [24, Proposition 3.11, pp. 60-61] to

$$
\widetilde{\mathcal{C}}^{s}\left(I^{*}\right)=\left\{f \in \mathcal{C}^{s}(\mathbb{R}): \operatorname{supp} f \subset[0,1 / 2]\right\}, \quad 0<s<2 l+1,
$$

$l \in \mathbb{N}_{0}$, where $I^{*}=(0,1 / 2)$. Any $f \in \widetilde{\mathcal{C}}^{s}\left(I^{*}\right)$ can be uniquely expanded by

$$
f(x)=\sum_{j \in \mathbb{N}} \sum_{k=1}^{2^{j}-1} f\left(2^{-j-1} k\right)\left(\sum_{m \in \mathbb{Z}} a_{k, m}^{l} v_{j, m}^{l}(x)\right)=\sum_{j \in \mathbb{N}} \sum_{m \in \mathbb{Z}} \lambda_{j, m} v_{j, m}^{l}(x), \quad x \in \mathbb{R},
$$

with

$$
\left\|f\left|\mathcal{C}^{s}(\mathbb{R}) \| \sim \sup _{j \in \mathbb{N}, m \in \mathbb{Z}} 2^{j s}\right| \lambda_{j, m} \mid\right.
$$


Furthermore $a_{k, m}^{l} \in \mathbb{R}$ and

$$
\left|a_{k, m}^{l}\right| \leq c^{\prime} 2^{-\varrho|2 m-k|}, \quad m \in \mathbb{Z}, \quad k \in \mathbb{N},
$$

for some $c^{\prime}>0, \varrho>0$, and

$$
\lambda_{j, m}=\sum_{k=1}^{2^{j}-1} a_{k, m} f\left(2^{-j-1} k\right) .
$$

Of course one can replace $I^{*}=(0,1 / 2)$ in $(3.16)$ by $I=(0,1)$ as needed in 3.3$)-(3.6)$. But this is an immaterial technical matter and we wanted to stick at the presentation given in [24].

3.3. Main assertion. In Hypothesis 3.1 we tried to compress the essence of the LengyelEpstein system displaying laminar Turing patterns. We wish to say something about the size of these patterns (estimates from below). For this purpose we rely on expansions in terms of Faber splines as described in Section 3.2 now extended to functions belonging to $\widetilde{\mathcal{C}}^{s}(Q)$.

TheOREM 3.3. Let $Q=(0,1)^{2}$ and $s>0$. Then there is a constant $c^{\prime}>0$ such that

$$
\begin{aligned}
& \int_{Q} u_{0}(x) \mathrm{d} x \leq c^{\prime} 2^{-J s}\left(\left\|u_{0} \mid \mathcal{C}^{s}(Q)\right\|+T\right), \\
& \int_{Q} v_{0}(x) \mathrm{d} x \leq c^{\prime} 2^{-J s}\left(\left\|v_{0} \mid \mathcal{C}^{s}(Q)\right\|+T\right),
\end{aligned}
$$

for all $J \in \mathbb{N}$, all $T>0$ and all functions $u_{0}, v_{0}$ and $u$, $v$ satisfying Hypothesis 3.1.

Proof. One can replace $Q=(0,1)^{2}$ by $(0,1 / 2)^{2}$ underlying the Faber expansions as described in Section 3.2 Let $0<s<2 l+1, l \in \mathbb{N}_{0}$. Then one has by (3.13), 3.17), (3.20)

$$
u\left(x_{1}, x_{2}, T\right)=\sum_{j \geq J} \sum_{m \in \mathbb{Z}} \lambda_{j, m} v_{j, m}^{l}\left(x_{1}\right)
$$

with

$$
\lambda_{j, m}=\sum_{k=1}^{2^{j}-1} a_{k, m}^{l} u\left(2^{-j-1} k, x_{2}, T\right), \quad j \geq J, \quad m \in \mathbb{Z},
$$

and 3.19 . We split the summation over $m \in \mathbb{Z}$ in 3.23 into terms with $|m| \leq 2^{j}$ and with $|m|>2^{j}$. By (3.14), 3.15) and (3.18) one has

$$
\begin{aligned}
\left|\int_{\mathbb{R}} \sum_{j \geq J} \sum_{|m| \leq 2^{j}} \lambda_{j, m} v_{j, m}^{l}\left(x_{1}\right) \mathrm{d} x_{1}\right| & \leq c^{\prime} \sum_{j \geq J} \sup _{m \in \mathbb{Z}}\left|\lambda_{j, m}\right| \\
& \leq c^{\prime \prime} 2^{-J s}\left\|u\left(\cdot, x_{2}, T\right) \mid \mathcal{C}^{s}(\mathbb{R})\right\| .
\end{aligned}
$$

If $k=1, \ldots, 2^{j}-1$ and $|m|>2^{j}$ then it follows from $|2 m-k| \sim|m|,(3.19)$ and (3.24) that

$$
\left|\lambda_{j, m}\right| \leq\left\|u\left(\cdot, x_{2}, T\right)\left|L_{\infty}(\mathbb{R})\left\|\sum_{k=1}^{2^{j}-1}\left|a_{k, m}^{l}\right| \leq c^{\prime}\right\| u\left(\cdot, x_{2}, T\right)\right| \mathcal{C}^{s}(\mathbb{R})\right\| 2^{j} 2^{-\varrho|m|}
$$


and, using again 3.15 ,

$$
\begin{aligned}
\left|\int_{\mathbb{R}} \sum_{j \geq J} \sum_{|m|>2^{j}} \lambda_{j, m} v_{j, m}^{l}\left(x_{1}\right) \mathrm{d} x_{1}\right| & \leq c^{\prime}\left\|u\left(\cdot, x_{2}, T\right) \mid \mathcal{C}^{s}(\mathbb{R})\right\| \sum_{j \geq J} \sum_{|m|>2^{j}} 2^{-\varrho|m|} \\
& \leq c_{s} 2^{-J s}\left\|u\left(\cdot, x_{2}, T\right) \mid \mathcal{C}^{s}(\mathbb{R})\right\| .
\end{aligned}
$$

By 3.23, 3.25, 3.27 and 3.5 one has

$$
\int_{Q} u(x, T) \mathrm{d} x \leq c^{\prime} 2^{-J s}\left\|u(\cdot, T) \mid \mathcal{C}^{s}\left(\mathbb{R}^{2}\right)\right\| .
$$

Similarly for $v\left(x_{1}, x_{2}, T\right)$. Then (3.21), 3.22 follow from Hypothesis 3.1

REMARK 3.4. We return to the discussion in Remark 3.2 Let $\delta=2^{-J}$ be the distance of the laminar vertical patterns as mentioned there. Then one has by (3.21)

$$
\delta \geq c^{\prime}\left(\frac{\int_{Q} u_{0}(x) \mathrm{d} x}{\left\|u_{0} \mid \mathcal{C}^{s}(Q)\right\|+T}\right)^{1 / s} .
$$

If the initial data $u_{0}$ are distributed rather evenly and if they vary only slowly than $\left\|u_{0} \mid \mathcal{C}^{s}(Q)\right\|$ differs from $\int_{Q} u_{0}(x) \mathrm{d} x$ not very much and $\delta$ is comparatively large. But if $u_{0}(x)$ is unevenly distributed and heavily oscillating than $\left\|u_{0} \mid \mathcal{C}^{s}(Q)\right\|$ might be large and $\delta$ might be small. These effects are confirmed by numerical simulation of equations underlying pattern formation as described in [11, 13] (and some other papers mentioned in Section 1). It supports the observation, also briefly mentioned at the end of Section 1 . that spots and stripes (governed by the Turing formalism) cannot be too small, in dependence on the oscillations of the initial data. Even Turing's original vision as quoted at the beginning of Section 1 fits in this scheme: One may assume that $u_{0}(x), v_{0}(x)$ are rather uniformly distributed, but randomly perturbed by small sharp peaks which do not contribute to $\int_{Q} u_{0}(x) \mathrm{d} x$ and $\int_{Q} v_{0}(x) \mathrm{d} x$ very much. But $\left\|u_{0} \mid \mathcal{C}^{s}(Q)\right\|$ and $\left\|v_{0} \mid \mathcal{C}^{s}(Q)\right\|$ may increase substantially having a strong impact on $\delta$ in (3.29).

3.4. Peaks, troughs, stripes, spots and Faber systems. This is (almost) the same heading as of [28, Section 5.6]. There we suggested to deal with specific Faber devices, originating from the classical Faber hat function in one dimension and their products in higher dimensions, as a reliable scheme to describe peaks, troughs, stripes, spots and other filigree structures as occur in chemotaxis and elsewhere, including Turing patterns. But this did not work as hoped beyond the very first comments in [28]. Now we return to this topic. But the present approach will be different. Roughly speaking we wish to make the assumptions underlying Theorem 3.3 and the discussions in Remark 3.4 more flexible.

We rely on the theory of the so-called refined localization spaces $F_{p, q}^{s, \text { rloc }}(\Omega)$ on $E$-thick domains $\Omega$ in $\mathbb{R}^{n}$. Although only the special case $F_{\infty, \infty}^{s}=B_{\infty, \infty}^{s}=\mathcal{C}^{s}$ in planar bounded domains $\Omega$ will be of interest for us later on, it seems to be reasonable to give a description of some crucial properties in terms of the general spaces $F_{p, q}^{s}$. A reader who is not familiar with the spaces $F_{p, q}^{s}$ may specify what follows by $F_{\infty, \infty}^{s}=\mathcal{C}^{s}$, what may justify that we do not repeat the basic definitions of $F_{p, q}^{s}$.

Let $l(Q)$ be the side-length of a (finite) open cube $Q$ in $\mathbb{R}^{n}$ with sides parallel to the axes of coordinates. A domain (= open set) $\Omega$ in $\mathbb{R}^{n}, n \in \mathbb{N}$, with $\Omega \neq \mathbb{R}^{n}$ is said to be 
E-thick (exterior thick) if one finds for any interior cube $Q^{i} \subset \Omega$ with

$$
l\left(Q^{i}\right) \sim 2^{-j}, \quad \operatorname{dist}\left(Q^{i}, \partial \Omega\right) \sim 2^{-j}, \quad j \geq j_{0} \in \mathbb{N},
$$

a complementing exterior cube $Q^{e} \subset \mathbb{R}^{n} \backslash \Omega$ with

$$
l\left(Q^{e}\right) \sim 2^{-j}, \quad \operatorname{dist}\left(Q^{e}, \partial \Omega\right) \sim \operatorname{dist}\left(Q^{i}, Q^{e}\right) \sim 2^{-j}, \quad j \geq j_{0} \in \mathbb{N} .
$$

Details and discussions may be found in [22, Section 3.1, pp. 69-77]. In particular, any bounded Lipschitz domain $\Omega$ in $\mathbb{R}^{n}$ is $E$-thick. Furthermore, the classical snowflake domain $\Omega$ in the plane $\mathbb{R}^{2}$ is $E$-thick, [22, Proposition 3.8, p. 75]. Let $\Omega$ be an $E$-thick domain in $\mathbb{R}^{n}$ and let

$$
0<p \leq \infty, \quad 0<q \leq \infty, \quad s>n\left(\max \left(\frac{1}{p}, \frac{1}{q}, 1\right)-1\right)
$$

$(q=\infty$ if $p=\infty)$. Then

$$
F_{p, q}^{s, \text { rloc }}(\Omega)=\widetilde{F}_{p, q}^{s}(\Omega)=\left\{f \in F_{p, q}^{s}\left(\mathbb{R}^{n}\right): \operatorname{supp} f \subset \bar{\Omega}\right\}
$$

with $F_{\infty, \infty}^{s}=B_{\infty, \infty}^{s}=\mathcal{C}^{s}$, [22, Proposition 3.10, pp. 77-78]. Here $F_{p, q}^{s, \text { rloc }}(\Omega)$ are the socalled refined localization spaces as introduced in [22, Section 2.2.3, pp. 36-40]. We recall the basic construction adapted to our needs. Let $\Omega$ be an arbitrary bounded domain (= open set) in $\mathbb{R}^{n}$ and let

$$
Q_{l, r}^{-1} \subset Q_{l, r}^{0} \subset Q_{l, r}^{1}, \quad l \in \mathbb{N} \text { and } r=1,2, \ldots,
$$

be concentric (open) cubes in $\mathbb{R}^{n}$ with sides parallel to the axes of coordinates, centered at $2^{-l} m^{r}$ for some $m^{r} \in \mathbb{Z}^{n}$ with the respective side-lengths $2^{-l-1}, 2^{-l}, 2^{-l+1}$ such that the cubes $Q_{l, r}^{0}$ are pairwise disjoint,

$$
\Omega=\bigcup_{l, r} \overline{Q_{l, r}^{0}} \quad \text { and } \quad \operatorname{dist}\left(Q_{l, r}^{1}, \partial \Omega\right) \sim 2^{-l}, \quad l \in \mathbb{N} .
$$

Details and references about this so-called Whitney decomposition may be found in [22, Section 2.1.2, pp. 30-31]. Let $\varrho=\left\{\varrho_{l, r}\right\}$ be a related resolution of unity consisting of non-negative functions with

$$
\operatorname{supp} \varrho_{l, r} \subset Q_{l, r}^{1}, \quad\left|D^{\gamma} \varrho_{l, r}(x)\right| \leq c_{\gamma} 2^{l|\gamma|}, \quad x \in \Omega, \quad \gamma \in \mathbb{N}_{0}^{n},
$$

for some $c_{\gamma}>0$,

$$
\sum_{l=1}^{\infty} \sum_{r} \varrho_{l, r}(x)=1 \quad \text { if } \quad x \in \Omega
$$

and

$$
\varrho_{l, r}(x)=1 \quad \text { if } \quad x \in Q_{l, r}^{-1} .
$$

For $p, q, s$ as in 3.32 we introduced in [22, Definition 2.14, pp. 36-37] the refined localization spaces

$$
F_{p, q}^{s, \text { rloc }}(\Omega)=\left\{f \in D^{\prime}(\Omega):\left\|f \mid F_{p, q}^{s, \text { rloc }}(\Omega)\right\|_{\varrho}<\infty\right\}
$$

with

$$
\left\|f \mid F_{p, q}^{s, \text { rloc }}(\Omega)\right\|_{\varrho}=\left(\sum_{l=1}^{\infty} \sum_{r}\left\|\varrho_{l, r} f \mid F_{p, q}^{s}\left(\mathbb{R}^{n}\right)\right\|^{p}\right)^{1 / p}
$$


(natural modification if $p=q=\infty$ ). They are quasi-Banach spaces which are independent of $\varrho=\left\{\varrho_{l, r}\right\}$ (equivalent quasi-norms), [22, Theorem 2.16, p. 37]. This assertion is based on related pointwise multiplier properties and the local homogeneity

$$
\left\|g_{l, r}\left|F_{p, q}^{s}\left(\mathbb{R}^{n}\right)\left\|\sim 2^{-l(s-n / p)}\right\| \varrho_{l, r} f\right| F_{p, q}^{s}\left(\mathbb{R}^{n}\right)\right\|
$$

where

$$
g_{l, r}(x)=\left(\varrho_{l, r} f\right)\left(2^{-l} m^{r}+2^{-l} x\right), \quad x \in \mathbb{R}^{n},
$$

normalizes the functions $\varrho_{l, r} f$ based now on the cubes $Q^{-1}, Q^{0}, Q^{1}$ centered at the origin and having respective side-lengths $1 / 2,1,2$. We refer the reader to [22, Theorem 2.11, Remark 2.12, pp. 34-35] and also to [27, Corollary 3.55, p. 116] for an improvement (not needed here).

We are interested here in planar bounded $E$-thick domains $\Omega$ in $\mathbb{R}^{2}$ such that its boundary

$$
\partial \Omega \quad \text { is a } d \text {-set with } 1 \leq d<2 .
$$

As usual, a compact set in $\mathbb{R}^{n}$ is called a $d$-set, $0 \leq d \leq n$, if it is the support of a Hausdorff $d$-measure. Recall that $d \geq 1$ is necessary for boundaries $\partial \Omega$ being a $d$-set for $E$-thick domains $\Omega$ in $\mathbb{R}^{2},[22$, Proposition 3.18, p. 86] where one finds also further explanations. In particular one needs in 3.35

$$
M_{l} \sim 2^{l d} \quad \text { squares } Q_{l, r}^{0}, \quad l \in \mathbb{N} .
$$

(A few related comments may be found in [22, p. 222].) We specify (3.33) to

$$
\mathcal{C}^{s, \text { rloc }}(\Omega)=\widetilde{\mathcal{C}}^{s}(\Omega)=\left\{f \in \mathcal{C}^{s}\left(\mathbb{R}^{2}\right): \operatorname{supp} f \subset \bar{\Omega}\right\}
$$

with $s>0$ and

$$
\left\|f\left|\widetilde{\mathcal{C}}^{s}(\Omega)\left\|\sim \sup _{l \in \mathbb{N} ; r=1, \ldots, M_{l}}\right\| \varrho_{l, r} f\right| \mathcal{C}^{s}\left(\mathbb{R}^{2}\right)\right\|, \quad f \in \widetilde{\mathcal{C}}^{s}(\Omega),
$$

according to 3.40 .

We wish to extend Theorem 3.3 to bounded $E$-thick domains $\Omega$ in $\mathbb{R}^{2}$ satisfying 3.43$)$. Let $\psi(x)=\left(\psi_{1}(x), \psi_{2}(x)\right), x \in \mathbb{R}^{2}$, be a diffeomorphic map of $\mathbb{R}^{2}$ onto itself. It is well known that not only $L_{1}\left(\mathbb{R}^{2}\right)$ but also all spaces $A_{p, q}^{s}\left(\mathbb{R}^{2}\right)$ are invariant with respect to diffeomorphic distortions $f(\psi(x))=(f \circ \psi)(x)$, [18, Theorem 4.3.2, p. 209]. This shows that one can replace in Theorem 3.3 the line segments 3.13 by suitable curved lines (including rotations).

Let $u(x, T) \in \widetilde{\mathcal{C}}^{s}(\Omega), s>0$. Then

$$
\int_{\Omega} u(x, T) \mathrm{d} x=\sum_{l=1}^{\infty} \sum_{r=1}^{M_{l}} \int_{\Omega} \varrho_{l, r}(x) u(x, T) \mathrm{d} x,
$$

where we used (3.37), 3.44). We rely on the reduction to the standard situation according to (3.41), 3.42 with $f(x)=u(x, T)$, apply 3.28 with $J-l$ in place of $J($ if $l<J)$, based on a suitable version of (3.13) with $J$ inside $Q_{l, r}^{1}$, and return to the squares in (3.34). Then one obtains

$$
\int_{Q_{l, r}^{1}} \varrho_{l, r}(x) u(x, T) \mathrm{d} x \leq c 2^{-2 l} 2^{-J s}\left\|\varrho_{l, r}(\cdot) u(\cdot, T) \mid \mathcal{C}^{s}\left(\mathbb{R}^{2}\right)\right\| .
$$


By using 3.46 and 3.44 it follows from 3.47 that

$$
\begin{aligned}
\int_{\Omega} u(x, T) \mathrm{d} x & \leq c^{\prime} 2^{-J s}\left\|u(\cdot, T) \mid \mathcal{C}^{s}\left(\mathbb{R}^{2}\right)\right\| \sum_{l=1}^{\infty} 2^{-l(2-d)} \\
& \leq c^{\prime \prime} 2^{-J s}\left\|u(\cdot, T) \mid \mathcal{C}^{s}\left(\mathbb{R}^{2}\right)\right\|
\end{aligned}
$$

(incorporating the terms with $l \geq J$, where no patterns are assumed). As far as the reduction of 3.49 and an $v(x, T)$-counterpart to assertions of type (3.21, 3.22 is concerned one must have in mind that on the one hand the created (straight or curved) lines in the squares $Q_{l, r}^{1}$ (originated by $\varrho_{l, r} u, \varrho_{l, r} v$ ) are independent to each other but on the other hand there is some overlap with exception of the spotted subdomain

$$
\Omega^{\circ}=\bigcup_{l=1}^{\infty} \bigcup_{r=1}^{M_{l}} Q_{l, r}^{-1},
$$

This follows from 3.34 and 3.37, 3.38 making clear that in $Q_{l, r}^{-1}$ only the related function $\varrho_{l, r}$ is different from zero. In other words, the above arguments produce spotted patterns on $\Omega$ with (independent curved) lines inside, having a minimal distance of type (3.29 from each other. This can be interpreted as coat patterns of animals (leopard etc.) or as the outcome of struggling chemicals in connection with CIMA chemical reactions based (observed experimentally and by numerical simulation) on (1.1), 1.2). In any case it supports the opinion that spots resulting from the Turing formalism cannot be too small, [1], Section 3.1, Mammalian Coat Patterns - How the Leopard Got Its Spots, pp. 142-156].

Acknowledgement. I wish to thank the referee for careful reading, valuable comments and suggestions.

\section{References}

[1] S. Abdelmalek, S. Bendoukha, B. Rebiai, On the stability and nonexistence of Turing patterns for the generalized Lengyel-Epstein model, Math. Methods Appl. Sci. 40 (2017), 62956305.

[2] V. Castets, E. Dulos, J. Boissonade, P. De Kepper, Experimental evidence of a sustained Turing-type equilibrium pattern, Phys. Rev. Lett. 64 (1990), 2953-2956.

[3] D. E. Edmunds, H. Triebel, Function Spaces, Entropy Numbers, Differential Operators, Cambridge Tracts in Math. 120, Cambridge Univ. Press, Cambridge, 1996.

[4] G. Gambino, M. C. Lombardo, M. Sammartino, Turing instability and pattern formation for the Lengyel-Epstein system with nonlinear diffusion, Acta Appl. Math. 132 (2014), 283-294.

[5] D. D. Haroske, H. Triebel, Entropy numbers in weighted function spaces and eigenvalue distributions of some degenerate pseudo-differential operators I, Math. Nachr. 167 (1994), 131-156.

[6] I. Lengyel, I. R. Epstein, Modeling of Turing structures in the chlorite-iodide-malonic acidstarch reaction systems, Science 251 (1991), 650-652.

[7] I. Lengyel, I. R. Epstein, A chemical approach to designing Turing patterns in reactiondiffusion systems, Proc. Natl. Acad. Sci. USA 89 (1992), 3977-3979. 
[8] B. Lisena, On the global dynamics of the Lengyel-Epstein system, Appl. Math. Comput. 249 (2014), 67-75.

[9] M. Ma, J. Tao, D. Wu, Y. Han, Non-constant steady states for the Lengyel-Epstein system with the CIMA reaction, Appl. Math. Lett. 30 (2014), 19-23.

[10] D. Mansouri, S. Abdelmalek, S. Bendoukha, On the asymptotic stability of the time-fractional Lengyel-Epstein system, Comput. Math. Appl. 78 (2019), 1415-1430.

[11] J. D. Murray, Mathematical Biology. II: Spatial Models and Biomedical Applications, third ed., Interdiscip. Appl. Math. 18, Springer, New York, 2003.

[12] W.-M. Ni, M. Tang, Turing patterns in the Lengyel-Epstein system for the CIMA reaction, Trans. Amer. Math. Soc. 357 (2005), 3953-3969.

[13] B. Perthame, Parabolic Equations in Biology, Lect. Notes Math. Model. Life Sci., Springer, Cham, 2015.

[14] W. Sickel, H. Triebel, Hölder inequalities and sharp embeddings in function spaces of $B_{p q}^{s}$ and $F_{p q}^{s}$ type, Z. Anal. Anwendungen 14 (1995), 105-140.

[15] A. Stevens, Mathematics and the life-sciences: A personal point of view, Jahresber. Dtsch. Math.-Ver. 119 (2017), 143-168.

[16] H. Triebel, Interpolation Theory, Function Spaces, Differential Operators, North-Holland, Amsterdam, 1978 (Sec. ed. Barth, Leipzig, 1995).

[17] H. Triebel, Theory of Function Spaces, Monogr. Math. 78, Birkhäuser, Basel, 1983.

[18] H. Triebel, Theory of Function Spaces II, Monogr. Math. 84, Birkhäuser, Basel, 1992.

[19] H. Triebel, Higher Analysis, Barth, Leipzig, 1992.

[20] H. Triebel, The Structure of Functions, Monogr. Math. 97, Birkhäuser, Basel, 2001.

[21] H. Triebel, Theory of Function Spaces III, Monogr. Math. 100, Birkhäuser, Basel, 2006.

[22] H. Triebel, Function Spaces and Wavelets on Domains, EMS Tracts in Math. 7, European Math. Soc., Zürich, 2008.

[23] H. Triebel, Bases in Function Spaces, Sampling, Discrepancy, Numerical Integration, EMS Tracts in Math. 11, European Math. Soc., Zürich, 2010.

[24] H. Triebel, Faber Systems and their Use in Sampling, Discrepancy, Numerical Integration, EMS Ser. Lect. Math., European Math. Soc., Zürich, 2012.

[25] H. Triebel, Local Function Spaces, Heat and Navier-Stokes Equations, EMS Tracts in Math. 20, European Math. Soc., Zürich, 2013.

[26] H. Triebel, Hybrid Function Spaces, Heat and Navier-Stokes Equations, EMS Tracts in Math. 24, European Math. Soc., Zürich, 2014.

[27] H. Triebel, Tempered Homogeneous Function Spaces, EMS Ser. Lect. Math., European Math. Soc., Zürich, 2015.

[28] H. Triebel, PDE Models for Chemotaxis and Hydrodynamics in Supercritical Function Spaces, EMS Ser. Lect. Math., European Math. Soc., Zürich, 2017.

[29] H. Triebel, Function spaces with dominating mixed smoothness, EMS Ser. Lect. Math., European Math. Soc., Zürich, 2019.

[30] A. M. Turing, The chemical basis of morphogenesis, Phil. Trans. Roy. Soc. London Ser. B 237 (1952), 37-72.

[31] L. Wang, H. Zhao, Hopf bifurcation and Turing instability of 2-D Lengyel-Epstein system with reaction-diffusion terms, Appl. Math. Comput. 219 (2013), 9229-9244.

[32] F. Yi, J. Wei, J. Shi, Diffusion-driven instability and bifurcation in the Lengyel-Epstein system, Nonlinear Anal. Real World Appl. 9 (2008), 1038-1051.

[33] F. Yi, J. Wei, J. Shi, Global asymptotic behavior of the Lengyel-Epstein reaction-diffusion system, Appl. Math. Lett. 22 (2009), 52-55. 